\title{
Complete, closed bacterial genomes from microbiomes using nanopore sequencing
}

\author{
Eli L. Moss ${ }^{1,3}$, Dylan G. Maghini ${ }^{1,3}$ and Ami S. Bhatt ${ }^{1,2} \bowtie$
}

\begin{abstract}
Microbial genomes can be assembled from short-read sequencing data, but the assembly contiguity of these metagenome-assembled genomes is constrained by repeat elements. Correct assignment of genomic positions of repeats is crucial for understanding the effect of genome structure on genome function. We applied nanopore sequencing and our workflow, named Lathe, which incorporates long-read assembly and short-read error correction, to assemble closed bacterial genomes from complex microbiomes. We validated our approach with a synthetic mixture of 12 bacterial species. Seven genomes were completely assembled into single contigs and three genomes were assembled into four or fewer contigs. Next, we used our methods to analyze metagenomics data from 13 human stool samples. We assembled 20 circular genomes, including genomes of Prevotella copri and a candidate Cibiobacter sp. Despite the decreased nucleotide accuracy compared with alternative sequencing and assembly approaches, our methods improved assembly contiguity, allowing for investigation of the role of repeat elements in microbial function and adaptation.
\end{abstract}

De novo generation of finished metagenome-assembled genomes (MAGs) for bacteria and archaea is a longstanding goal in microbiome research. As existing metagenomic sequencing and assembly methods do not usually yield finished bacterial genome sequences, genome drafts are formed by grouping or 'binning' similar contigs. This approach has produced enormous collections of bacterial genomes and substantially expanded our appreciation of the microbial world ${ }^{1-4}$

Binning quality largely relies on the size and contiguity of the underlying assembly. As assembly contiguity increases, the sensitivity and specificity of genome binning are improved, because fewer, larger contigs need to be grouped to form each genome. Advances in sequencing and assembly technologies, including read-cloud sequencing, have improved MAG quality ${ }^{5}$, but remain limited in their ability to correctly place repeat sequences.

Repeat elements can range in size from tens of base pairs to hundreds of kilobases ${ }^{6}$. Long reads can span entire common repeat elements such as miniature inverted repeat transposable elements, transposons, gene duplications and prophage sequences. Recently, nanopore and PacBio long-read assembly methods have been applied to the gut and other microbiomes ${ }^{7,8}$. However, the application of long-read methods to analyze gut microbiomes has been hindered by the lack of efficient methods to extract high molecular weight (HMW) DNA from stool. Standard bead beating can result in extensive shearing, and although solid phase reversible immobilization (SPRI) bead 'cleanup' steps remove DNA fragments in the low hundreds of base pairs, this often fails to enrich for DNA fragments that are sufficiently large to scaffold across bacterial repeat elements. Gentle bead beating can reduce shearing, but might fail to extract DNA from organisms that are difficult to lyse. Thus, there is a need for methods to extract long fragments of DNA that can span repetitive elements from both Gram-positive and Gram-negative bacteria to overcome limitations in genome assembly.

We present a workflow for nanopore sequencing of stool samples, including protocols for DNA extraction and genome assembly (Supplementary Fig. 1). Our DNA extraction protocol is adapted from extraction methods for cultured bacteria ${ }^{9}$, and comprises enzymatic degradation of the cell wall with a cocktail of lytic enzymes, then phenol-chloroform extraction, followed by RNAse $\mathrm{A}$ and Proteinase $\mathrm{K}$ digestion, gravity column purification and SPRI size selection. This approach produces microgram quantities of pure, HMW DNA suitable for long-read sequencing from as little as $300 \mathrm{mg}$ of stool. Our bioinformatics workflow, Lathe, uses a long-read assembly based approach, rather than a hybrid assembly method such as OPERA-MS, which was recently reported ${ }^{8}$. Input long-read data can be generated by either nanopore or PacBio technologies. Lathe combines existing steps for basecalling, long-read assembly and polishing with refined approaches for misassembly detection and genome circularization (Methods).

We first sought to test whether we could assemble closed bacterial genomes by using a standard ATCC 12-species mixture (Methods) that comprises both Gram-positive and Gram-negative bacteria. Due to the low concentration of HMW DNA present in the lyophilized cell material provided and the reduced contamination in this synthetic mixture compared with stool samples, we omitted the digestion and gravity column steps for DNA extraction (Methods) and obtained $401 \mathrm{ng}$ of HMW DNA (Supplementary Table 1 and Supplementary Fig. 2). We used nanopore sequencing and obtained 30.3 gigabase pairs ( $\mathrm{Gbp}$ ) of long-read data with a read N50 of 5.9 kilobase pairs (kbp) (Supplementary Table 2) containing all 12 constituent species in approximately even relative abundances after correction for genome length (Fig. 1). Taxonomic classification of long reads demonstrated that read-length distributions vary between organisms (Fig. 1) from a minimum read N50 of $2.3 \mathrm{kbp}$ (Fusobacterium nucleatum) to a maximum of $8.5 \mathrm{kbp}$ (Bacteroides fragilis), perhaps a consequence of slight variations in response to lysis, extraction, lyophilization or storage. Gram-positive bacteria (red crosses, Fig. 1), which generally have a thicker peptidoglycan cell wall than Gram-negative bacteria, are not categorically depleted in relative abundance.

Assembly using Lathe yielded a total assembly N50 of $4.6 \mathrm{Mbp}$ and total length of $48 \mathrm{Mbp}$, in agreement with the known total reference length (Supplementary Table 3). Lathe achieved a higher assembly N50 than other long-read assembly tools (1.6- to fourfold improvement) and hybrid assembly tools (two- to nine-fold improvement) (Supplementary Table 4). By contrast, assembly with

'Department of Genetics, Stanford University, Stanford, CA, USA. '2Department of Medicine (Hematology, Blood and Marrow Transplantation), Stanford University, Stanford, CA, USA. ${ }^{3}$ These authors contributed equally: Eli L. Moss, Dylan G. Maghini. ${ }^{凶}$ e-mail: asbhatt@stanford.edu 
SPAdes ${ }^{10}$ of a $7.7 \mathrm{Gbp}$ public short-read dataset from the same mixture produces an assembly N50 of $133 \mathrm{kbp}$. Assembly with Lathe of nanopore data, randomly downsampled to equal the smaller total size of the short-read dataset, yielded an N50 of 3.3 Mbp, a 25-fold improvement over the short-read assembly.

Of the 12 bacteria in the mixture, seven were assembled into single contigs and shown to be complete by alignment to available closed reference sequences (Fig. 1). Three more genomes were assembled into four contigs or fewer. The most incomplete assembly contained $83 \%$ of the genome in a single contig. Our assembly contains large-scale inversions in Bacteroides vulgatus and Enterobacter cloacae relative to reference genome sequences. These were retained after multiple misassembly removal steps as the assembled inversion breakpoints were each spanned by multiple long reads. These inversions are flanked by homologous regions $>20 \mathrm{kbp}$ in length; this makes determining the true orientation of the inverted segment extremely challenging and, thus, we are unsure whether this represents an error in our assembly or the reference sequence. MetaQuast ${ }^{11}$ comparison of available closed reference genome sequences to our assemblies after consensus refinement with long reads, short reads or combined long and short reads indicates that short-read refinement is sufficient for error correction (Supplementary Note 1), but we have included the option of combining both forms of correction for cases of sparse short-read coverage in the Lathe workflow.

Next, we applied our methods to two human stool samples that were previously used to evaluate short-read and read-cloud sequencing and assembly approaches ${ }^{5}$, here referred to as samples P1 and P2-A, as well as a stool sample collected 15 months after the first sample from individual P2; we refer to this second sample as P2-B. Our extraction approach yielded at least $1 \mu \mathrm{g}$ of pure HMW DNA per $300 \mathrm{mg}$ of input stool mass for all samples (Supplementary Table 1). We tested the potential generalizability of the extraction approach on canine and murine stool samples and obtained similar yield, purity and fragment size across all samples tested (Supplementary Fig. 2).

After nanopore sequencing, we obtained a total of $12.7,6.1$ and 7.6 Gbp of long-read data for samples P1, P2-A and P2-B, respectively (Supplementary Table 2) with read N50 values of 4.7, 3.0 and $3.0 \mathrm{kbp}$ (Supplementary Table 2 and Supplementary Fig. 3). DNA from these samples was extracted before the incorporation of MetaPolyzyme into our approach, so it is possible that taxa that are difficult to lyse may be underrepresented. Nonetheless, taxonomic composition of reads obtained through this version of our approach had higher Shannon diversity when compared with reads from samples extracted with mechanical lysis and short-read sequencing (Fig. 2). Specifically, we recovered all genera detected by more than 200 short reads and there was no categorical depletion of typically lysis-resistant Gram-positive organisms (Supplementary Fig. 4). Per-species read-length distribution for organisms occurring in the natural samples was less variable than in the synthetic bacterial mixture (Supplementary Fig. 5). We observed a prominent 3-4kbp subset of reads classified as Escherichia coli in all samples and a second $12-15 \mathrm{kbp}$ subset of reads classified as Enterococcus faecalis in the defined bacterial mixture (Supplementary Fig. 5). These reads originate from sequences with high identity to phage and are not found in a PacBio library prepared from the same extraction, suggesting contamination of nanopore libraries with phage DNA.

Assembly with Lathe yielded whole-assembly N50 values of 236, 221 and $179 \mathrm{kbp}$ and total assembly sizes of 139,83 and $87 \mathrm{Mbp}$ for samples P1, P2-A and P2-B, respectively. Employing a strategy to improve metagenomic assembly of related communities ${ }^{12}$, we coassembled samples P2-A and P2-B and obtained a 1.7-fold increase in assembly $\mathrm{N} 50(384 \mathrm{kbp})$ and a 1.3-fold increase in total assembly size (112 Mbp) (Supplementary Table 5). In comparison, short-read assembly yielded assembly N50 values of 34 and $15 \mathrm{kbp}$ for P1 and $\mathrm{P} 2-\mathrm{A}$, in spite of a three- to six-fold higher input (total bases) of raw read data, and read-cloud assembly yielded N50 values of 116 and $12 \mathrm{kbp}$ in P1 and P2-A. However, read-cloud and short-read assemblies were between 1.5- and 2.1-fold larger in total than corresponding nanopore long-read assemblies, likely due to the much greater volume of raw data in these datasets (Supplementary Tables 2 and 5). Sequencing with PacBio produced an assembly that was much more fragmentary than that produced by nanopore sequencing and assembly with Lathe, likely due to more variable coverage with PacBio sequencing (Supplementary Note 2).

After binning contigs from nanopore, read-cloud and short-read approaches to form draft genomes ${ }^{5}$, drafts were scored as 'High Quality' or 'Partial' based on completeness, contamination, and presence of 5S, 16S, 23S ribosomal RNA and transfer RNA loci ${ }^{1}$. Completeness and contamination were assessed using check ${ }^{13}$, a tool that evaluates for presence of single-copy core genes; while broadly applied and useful, circumstances have been documented where estimates of completeness and contamination are inaccurate $^{14}$. The long-read approach produced bins with much higher contiguity than the read-cloud approach, at lower cost, (Fig. 2, Supplementary Fig. 6 and Supplementary Table 6), yielding several high-quality genomes with $\mathrm{N} 50$ over $2 \mathrm{Mbp}$, whereas the read-cloud approach yielded only one and the short-read approach yielded no bins with N50 values greater than $0.55 \mathrm{Mbp}$. Nanopore sequencing assembled several single-contig, high-quality genomes from each sample, including genomes for Dialister sp., Faecalibacterium prausnitzii, Oscillibacter sp. and P. faecium, all of which had fragmentary read-cloud and short-read assemblies (Fig. 2 and Supplementary Fig. 7). Notably, our approach produced a circular genome for $P$. copri, an organism that lacked a closed reference until recently ${ }^{7}$, in spite of extensive previous efforts to assemble it and other members of the genus ${ }^{15}$. Several bins in the $\mathrm{P} 1$ read-cloud assembly were absent from nanopore bins, likely due to their low coverage depth (3-40x), resistance to lysis or cooccurrence with closely related community members.

We then sought to evaluate the generalizability of our extraction and nanopore sequencing approach and to test whether this HMW DNA extraction approach generated taxonomically concordant results compared to conventional bead beating. With MetaPolyzyme incorporated in the lysis stage, we applied the two extraction approaches to ten additional stool samples from healthy adults (samples A-J). An adequate amount of size-selected DNA was obtained from all ten of the samples using the HMW DNA extraction approach. While we attempted to obtain sufficiently sizeselected DNA for nanopore sequencing from DNA extracted with bead beating for a subset of the stool samples, we were only able to obtain adequate DNA from one sample (Supplementary Fig. 8). Nanopore sequencing of the HMW extracted DNA yielded 13 to $27 \mathrm{Gbp}$ of raw long-read data with read N50 values ranging from 1.4 to $5.2 \mathrm{kbp}$, which was combined with comparatively light coverage of 1.9 to $3.6 \mathrm{Gbp}$ of short-read data for consensus refinement and determining taxonomic composition (Supplementary Table 2). Nanopore sequencing on the sample that had sufficient bead-beaten DNA yielded a read $\mathrm{N} 50$ of $2.5 \mathrm{kbp}$ and $6.3 \mathrm{Gbp}$ of data, compared to $2.7 \mathrm{kbp}$ and $15.9 \mathrm{Gbp}$ for nanopore sequencing of a HMW extraction on the same sample. Both extraction methods yielded similar taxonomic compositions when nanopore sequenced (Supplementary Fig. 9). Nanopore and short-read data were classified and compared across samples (Supplementary Figs. 10 and 11 and Supplementary Table 7). On log-transformed read counts of all 596,300 species classified, we measured an overall correlation (Pearson $r=0.79$ ) between the two approaches. The number of read counts ranged from 1 to $5.7 \times 10^{6}$, with a mean of 620 (Supplementary Fig. 12). Of the 18,642 instances of a ten-fold or greater difference in relative abundance of a particular species between the two approaches, our approach yielded the higher relative abundance in $95 \%$ of cases, suggesting the potential for greater taxonomic sensitivity by our method. 
a

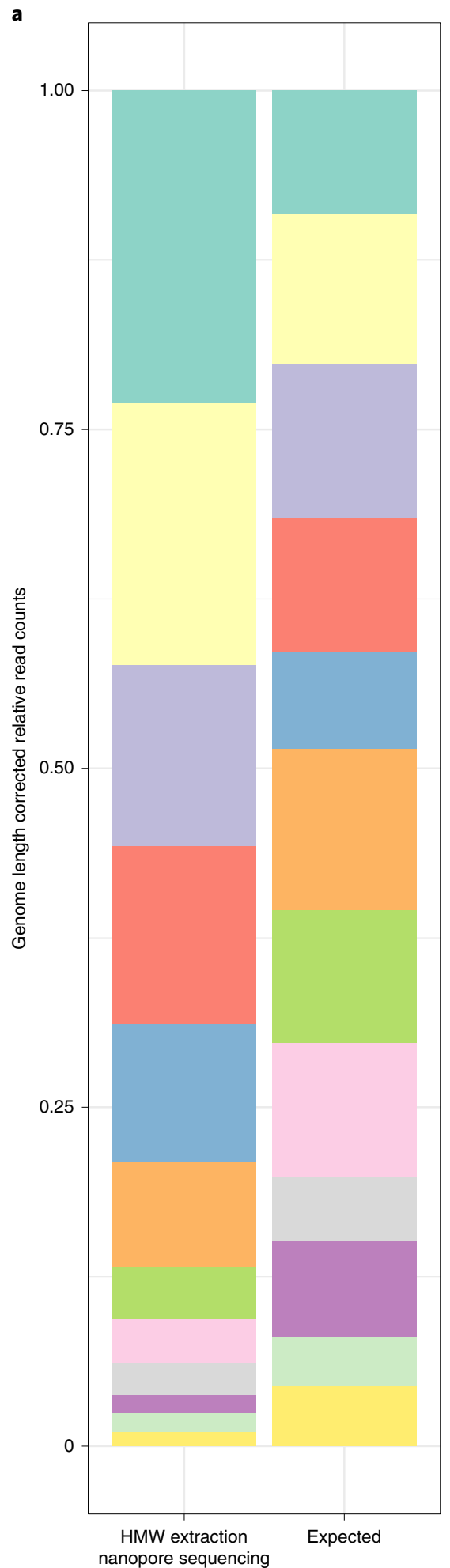

b

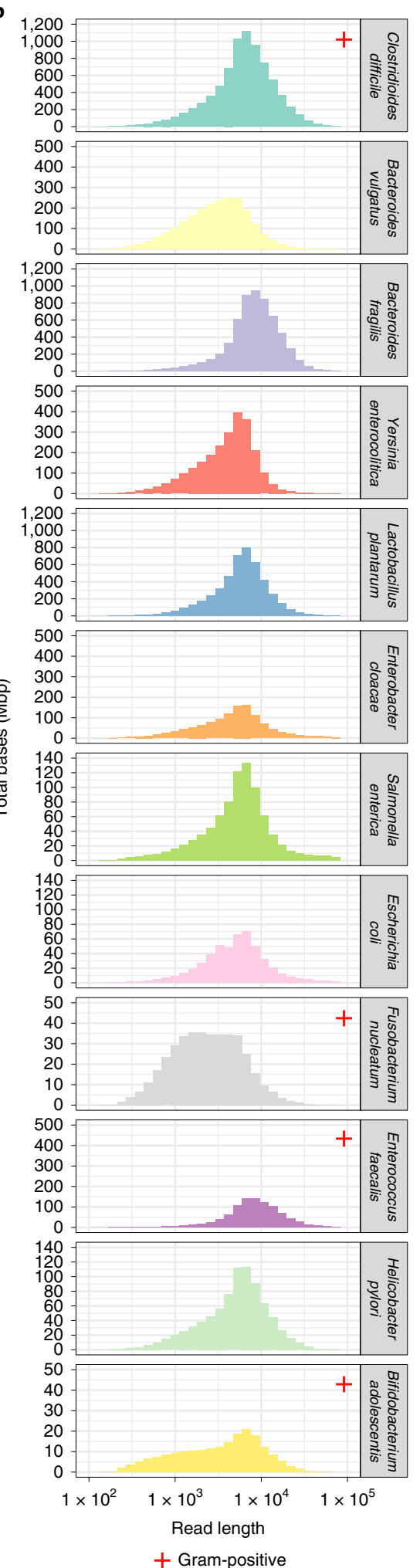

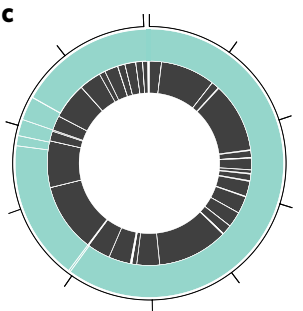
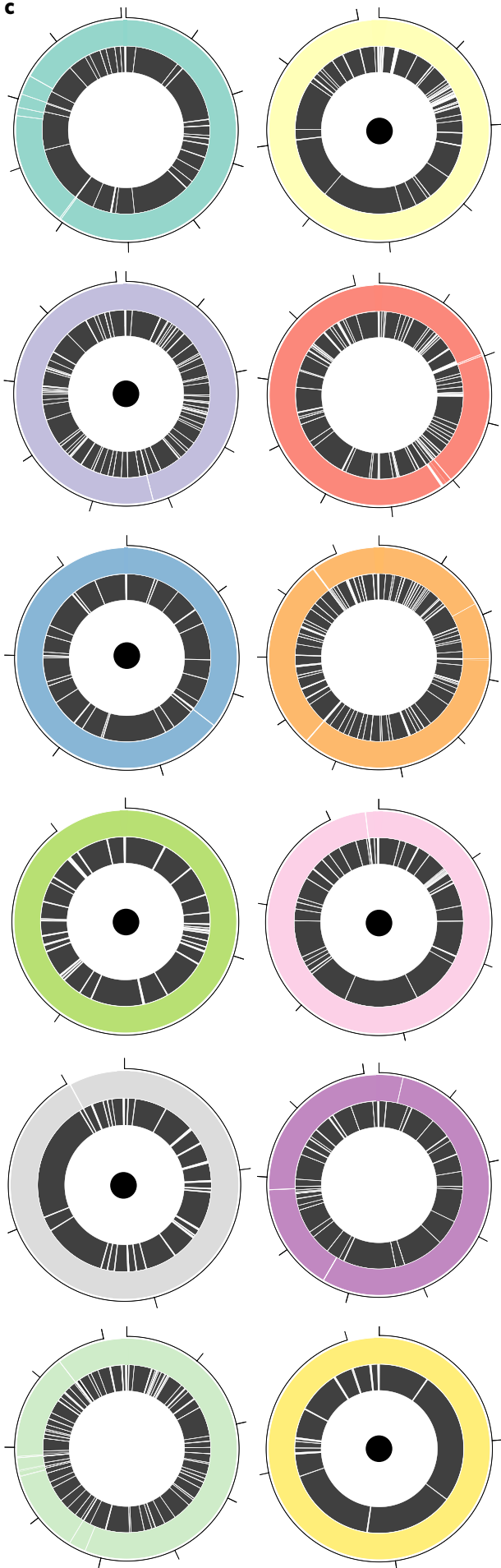

Single-contig assembly

Fig. 1 | Taxonomic read composition, per-organism read-length distributions and genome assemblies in a defined 12-species bacterial mixture.

a, Relative read counts are shown for the expected equal composition of bacterial cells and the observed composition, with correction for relative genome size. b, Read-length distributions per organism. Individual organisms demonstrate varying read-length distributions in some cases. c, Circos plots demonstrate the relative assembly contiguity of the nanopore versus short-read assembly approaches. Nanopore sequencing and assembly (colored outer ring) outperforms short-read assembly (black inner ring), producing complete genome assemblies (small black inner dots) in seven of 12 cases, with a further three assembled in four contigs or fewer. Numbers indicate genome size in megabases. Note that complete assemblies may contain one apparent break due to differing linearization breakpoints in reference and assembly sequences. 

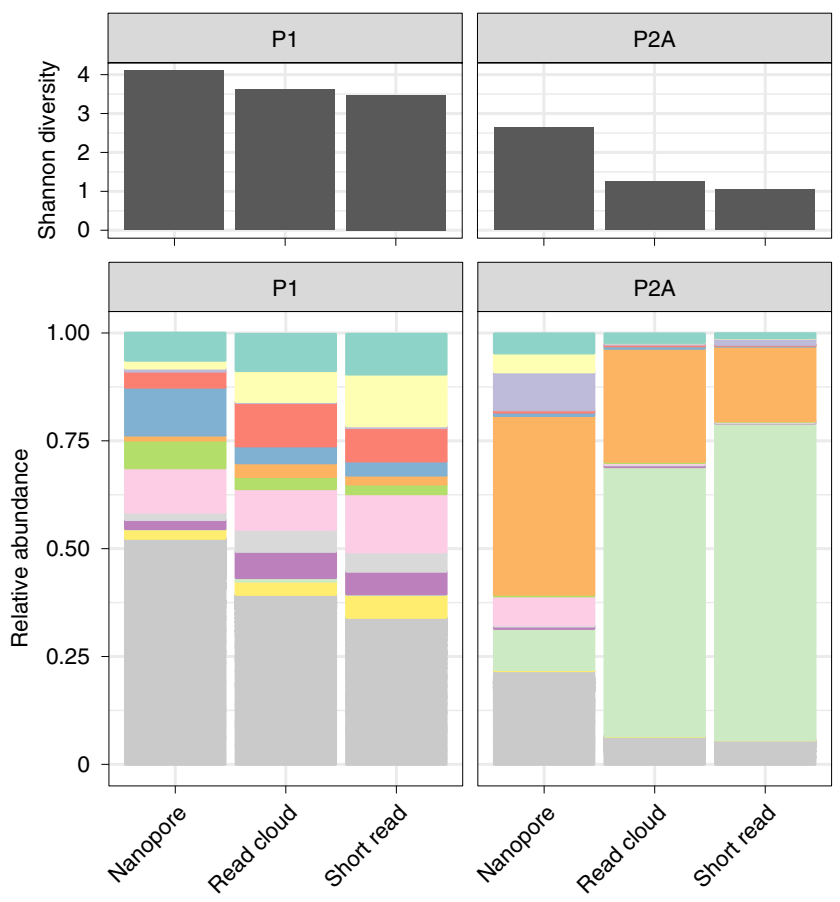

b

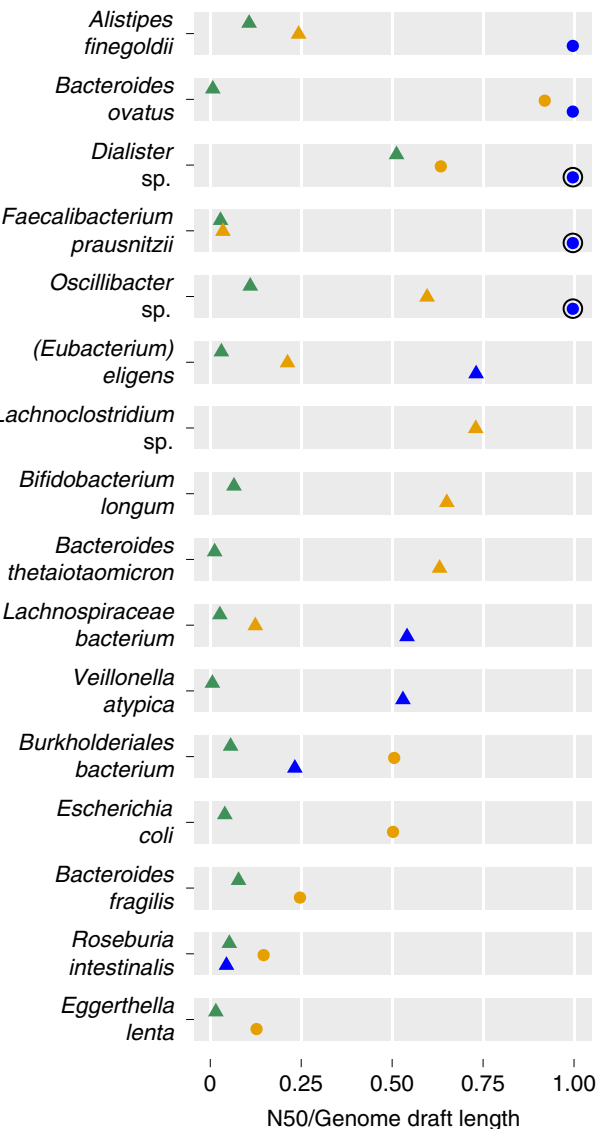

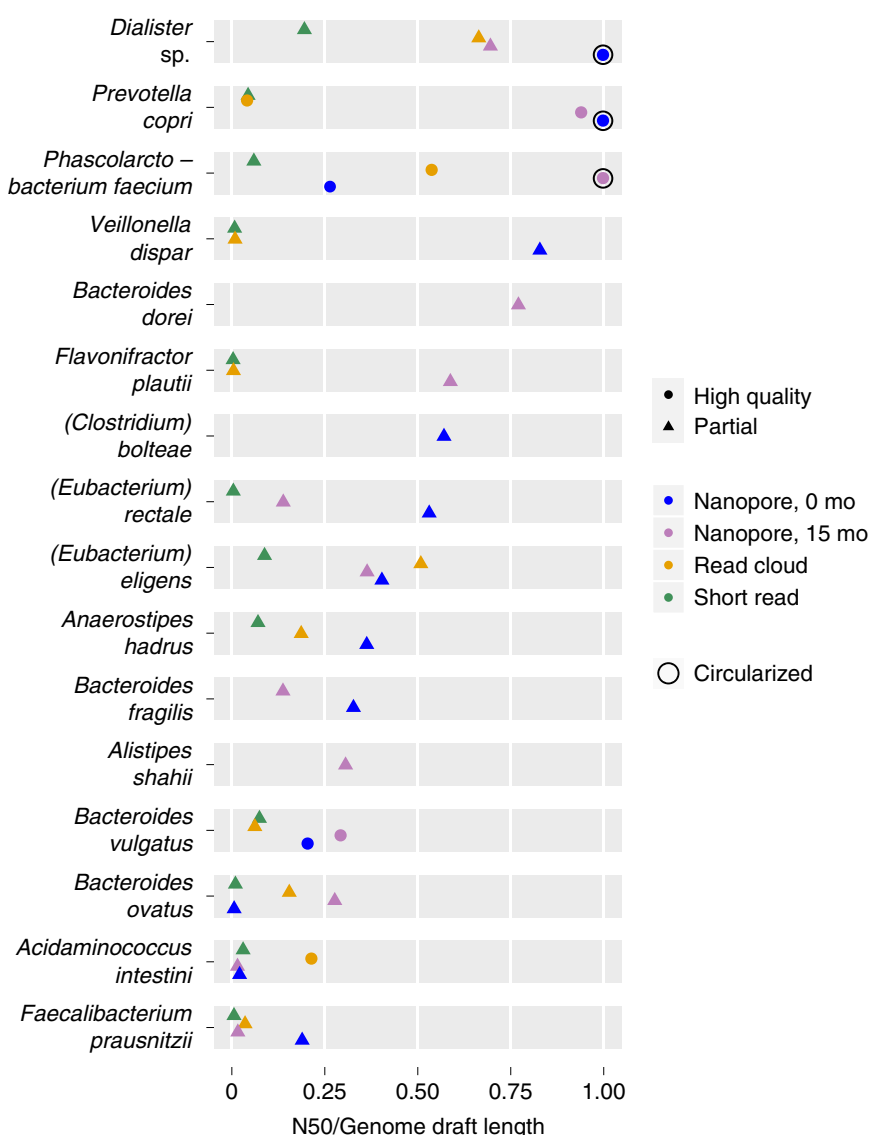

- High quality

Partial

- Nanopore, 0 mo

- Nanopore, 15 mo

- Read cloud

Circularized

(Eubacterium) eligens

(Eubacterium) rectale

Anaerostipes hadrus

Bacteroides fragilis

Bacteroides ovatus

Bacteroides vulgatus

Bacteroides xylanisolvens

Faecalibacterium prausnitzii

Parabacteroides distasonis

Parabacteroides sp. СТ06

Prevotella copri

Roseburia intestinalis

Other

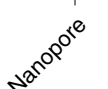

Fig. 2 | Per-organism assembly contiguity, diversity and taxonomic read composition in two healthy human stool microbiomes. a, Species-level Shannon diversity is shown for the sequence datasets obtained. Higher diversity is found in libraries prepared with the present DNA extraction method. Relative species-level abundances are shown for a conventional workflow consisting of bead-beating extraction and short-read sequencing, as well as the present workflow consisting of HMW DNA extraction and long-read sequencing. b. Contiguity is expressed as per-bin N50 divided by per-bin length (the total length of sequences assigned to the bin). As bin assembly approaches completion, the quantity N50 divided by bin length approaches one, regardless of genome size. Nanopore sequencing and assembly (blue, purple) demonstrates higher assembly contiguity than read-cloud (gold) and short-read (green) approaches. For all organisms achieving assembly N50 of at least $500 \mathrm{kbp}$ or a complete genome draft by any approach, genome draft quality and contiguity are shown for long reads, read clouds and short reads. Shapes indicate draft quality. Asterisk marks a genome later annotated as putative Cibiobacter. 
Table 1 | Circular bacterial genomes assembled from human stool samples

\begin{tabular}{|c|c|c|c|c|c|c|c|}
\hline Genome & Sample & Assembler & $\begin{array}{l}\text { Genome size } \\
\text { (Mbp) }\end{array}$ & Genes & $\begin{array}{l}\text { 16S } \\
\text { rRNA }\end{array}$ & GC percentage & Transposases $^{\mathrm{b}}$ \\
\hline Dialister sp. & P1 & Canu & 1.96 & 1,912 & 4 & 45.1 & 2 \\
\hline Dialister sp. & P2-A & Canu & 1.89 & 1,803 & 4 & 45.3 & 7 \\
\hline Faecalibacterium prausnitziic & P1 & Canu & 3.4 & 3,234 & 6 & 56.1 & 45 \\
\hline Oscillibacter sp. & P1 & Canu & 3.04 & 2,926 & 3 & 60.1 & 4 \\
\hline Phascolarctobacterium faecium & P2-B & Canu & 2.35 & 2,307 & 5 & 44.0 & 0 \\
\hline Akkermansia muciniphila & I & Flye & 3.01 & 2,906 & 3 & 55.2 & 0 \\
\hline Anaerotruncus sp. & $\mathrm{F}$ & Flye & 2.11 & 2,156 & 2 & 43.5 & 4 \\
\hline Bacteroides sp. & $\mathrm{F}$ & Flye & 3.04 & 2,467 & 3 & 48.9 & 18 \\
\hline Clostridales sp. ${ }^{a}$ & D & Flye & 2.05 & 1,971 & 2 & 53.2 & 0 \\
\hline Eubacterium siraeum ${ }^{a}$ & $\mathrm{~F}$ & Flye & 3.12 & 2,894 & 3 & 45.3 & 12 \\
\hline Phascolarctobacterium faecium & । & Flye & 2.35 & 2,481 & 5 & 43.4 & 0 \\
\hline Prevotella sp. & $\mathrm{F}$ & Flye & 3.46 & 3,031 & 5 & 45.8 & 78 \\
\hline Roseburia sp. ${ }^{a}$ & $\mathrm{D}$ & Flye & 2.17 & 2,953 & 2 & 40.9 & 8 \\
\hline Ruminococcus bromii & G & Flye & 2.21 & 2,820 & 3 & 40.7 & 20 \\
\hline Ruminococcus sp. & $\mathrm{E}$ & Flye & 2.48 & 3,007 & 4 & 42.1 & 0 \\
\hline Sellimonas intestinales ${ }^{\mathrm{a}}$ & $\mathrm{D}$ & Flye & 1.76 & 2,889 & 3 & 45.2 & 11 \\
\hline
\end{tabular}

${ }^{a}$ Assembly broken into multiple contigs during final misassembly detection, perhaps due to off-by-one circularization. ${ }^{\mathrm{b}}$ Transposases annotated with Prokka v.1.13.3. CLater annotated as putative Cibiobacter sp.

While 3,566 classifications made by the bead beating and short-read approach were undetected by the present approach, our approach made 72,989 classifications undetected by the bead-beating and short-read approach. Across the ten samples, we obtained assemblies ranging in total length between 48 and $207 \mathrm{Mbp}$ and assembly N50 between 51 and $120 \mathrm{kbp}$, the two generally inversely correlated (Supplementary Table 5). This is slightly reduced compared to the assemblies obtained from human stool samples P1 and P2, likely due to our use of the Flye assembler in place of Canu, incurring much lower computational cost in exchange for a modest reduction in contiguity (see Methods and Supplementary Table 4). In situations where high contiguity is desired, such as attempts to generate a complete, closed genome of a novel taxon or when sensitive detection of structural variants or horizontally transferred intrachromosomal genomic regions is desired, Canu may be the preferred assembler. Alternatively, when aiming to obtain as many high-quality genome bins as possible and cost is a higher priority consideration, Flye may be the preferred assembler.

This sequencing and assembly approach is capable of generating closed, circular genomes (Table 1). However, as we learned from our sequencing and assembly experiments with the mock mixtures, fully assembled genomes often evade circularization from their initial linear form. In the context of mock mixtures, the ground truth is known; however, in natural samples where a ground truth is lacking, it is difficult to determine whether genomes that do not circularize are truly 'full genomes' ${ }^{\text {'14. }}$.

For example, from the three stool samples that were assembled with Canu (P1, P2-A and P2-B), our approach yielded eight highquality, single-contig bacterial genomes and a maximum of five from a single sample (P1), compared to zero from short-read and read-cloud approaches ${ }^{5}$. Lathe achieved precise circularization for five of these genomes. Closed genomes ranged in coverage depth between $75 \times($ Oscillibacter sp.) and $785 \times($. copri), and were largely structurally concordant and similar in sequence to existing published genome sequences (Supplementary Fig. 7), although in some cases, we note extensive strain divergence. For example, our closed Dialister sp. genome exhibits multiple large-scale inversions relative to the available reference concordant with previous read-cloud and short-read assemblies ${ }^{5}$.

The circular P. copri genome (Fig. 3a) is especially notable, as our own previous attempts using read clouds, short reads and synthetic long reads to assemble these communities also had limited success with this organism, never exceeding a genome N50 of $130 \mathrm{kbp}$ in spite of attempts with coverage depth in excess of $4,800 \times$, as well as with downsampled datasets ${ }^{5}$. While this is a report of a $P$. copri genome from a human sample, recently the first single-contig $P$. copri genome was reported using a nanopore approach on cow rumen ${ }^{7}$, supporting the use of longer reads in solving these difficult assemblies. The difficulty of assembling the $P$. copri genome stems from its high degree of sequence repetition. Previous assembly of repetitive $k$-mers in $P$. copri identified five repeat sequences with high identity to known transposase sequences ${ }^{5}$, and additional annotation with Prokka ${ }^{16}$ reveals additional insertion sequence transposases. The location of these high copy number elements is resolved in our circular assembly, and often fall at the locations of breaks in previous short-read and read-cloud assemblies of $P$. copri.

Noting high strain divergence between our circular F. prausnitzii genome and available references, we attempted to improve classification using 16S rRNA gene classification. Top hits for all six $16 \mathrm{~S}$ rRNA sequences fell between Gemmiger formicilis (average of 98.11\% identity) and Subdoligranulum variabile (average of $98.19 \%$ identity), compared to only $92.63 \%$ identity with F. prausnitzii type strain $16 \mathrm{~S}$ rRNA sequences, indicating that this genome may be a member of the recently described Cibiobacter clade $^{17}$ and may represent a closed genome for this genus (Fig. 3b). We identified five 


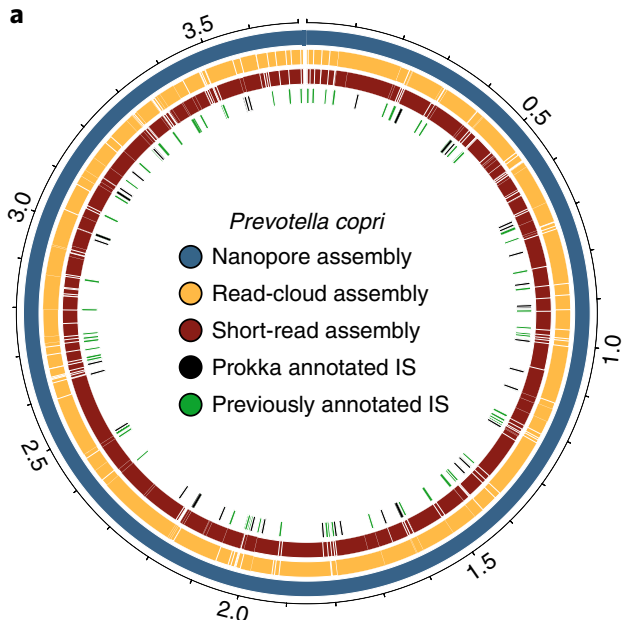

b

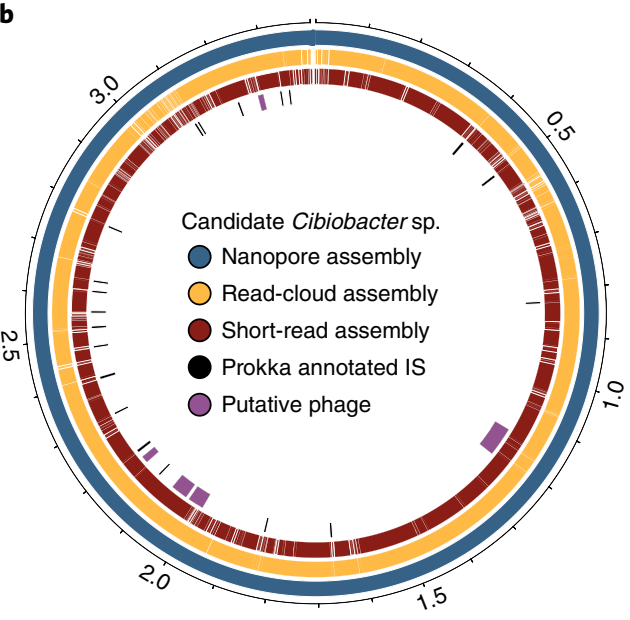

Fig. 3 | Circos diagrams of closed, circular genomes of $P$. copri and Cibiobacter sp. a, $P$. copri from P2-A. b, Cibiobacter sp. from P1. In both plots, the outermost ring represents the complete, closed and circularized genome of the given organism. The middle and inner rings represent contigs from the corresponding read-cloud and short-read assemblies, respectively, that were mapped to the nanopore assembly. The inner track in each case displays annotated, predicted mobile genetic elements such as insertion sequences (ISs), transposases and prophage.

putative phage in the closed assembly, ranging in length from 8.5 to $65.9 \mathrm{~kb}$.

Additionally, we obtained a total of 11 fully circularized, singlecontig genomes from the set of ten samples that were assembled with Flye (samples A-J, Table 1). Among these is another completed Prevotella genome belonging to a species closely related to CAG:386 (92\% draft aligned at $98 \%$ identity) representing a completed reference for this species. In total, we obtained 19 high-quality genomes, 16 of which had an N50 of over $1 \mathrm{Mbp}$ and 12 had N50 over $2 \mathrm{Mbp}$ (Supplementary Fig. 13). An additional 22 genomes met these criteria with minimum completeness reduced to $75 \%$. In total, 1,219 genome drafts with contamination $<5 \%$ were recovered, ranging in completeness from 0.31 to $100 \%$ with a mean of $23 \%$.

However, even well covered genomes can fail to assemble when they have high identity with other organisms in the community, as Lathe cannot construct an unambiguous representation of a single contiguous genome from a highly interconnected assembly graph (Supplementary Fig. 14). Additionally, assembly contiguity remains closely tied to the DNA fragment length, and while the high read lengths achieved by our approach improve assembly contiguity over short-read approaches, further improvement in extraction methods will be necessary to achieve the longer read lengths needed to fully resolve clusters of highly related genomes and longer structural variants. Our extraction method has been demonstrated to more reliably extract HMW DNA from samples than bead-beating approaches do, thus offering many advantages for completing and circularizing genomes, but it does have extraction biases for different bacterial species that will necessitate further investigation. Gentler bead-beating approaches may also yield HMW DNA, but at the expense of potentially failing to extract DNA from difficult to lyse organisms. Therefore, standard bead beating remains the best approach for accurately measuring relative abundances of taxa. As short-read polishing improves assembly quality (Supplementary Note 1), reads used for polishing can inform relative abundance.

In the past several years, assorted molecular and computational approaches have been described for generating more complete genomes from metagenomes. Read-cloud sequencing with Athena assembly is advantageous in situations where DNA is scarce, as the input requirement is $\sim 100$-fold lower than that required for standard long-read sequencing approaches. This can be particularly helpful when studying low biomass samples, such as clinical samples. At present, limited comparisons between hybrid assemblers and long-read assembly followed by short-read polishing have been made. Based on the concepts that underlie these two approaches, hybrid assembly may be preferred in situations where low-coverage long-read sequence data is available.

In conclusion, we anticipate that our approach will enable the mapping of horizontally transferred gene segments, such as prophage, into specific genomic contexts. This may help to illuminate how structural strain variation within the microbiome may link to microbial function $^{18}$. Furthermore, this approach enables the proper placement of repetitive genetic elements, as exist in the genus Prevotella, where such variations can be important in bacterial metabolic phenotypes ${ }^{19}$. Improved references in this group and elsewhere will facilitate study of diverse gut microbiomes across global populations by allowing investigation into the complete functional repertoire and potential phenotypes of individual microbes, even when these organisms are difficult to culture or occur in complex communities. We expect that advances in metagenomic DNA extraction methods, long-read sequencing, assembly algorithms and epigenetic modification detection ${ }^{20}$ will further improve the quality of MAGs, causing a profound shift in the effectiveness and resolution of metagenomic assembly.

\section{Online content}

Any methods, additional references, Nature Research reporting summaries, source data, extended data, supplementary information, acknowledgements, peer review information; details of author contributions and competing interests; and statements of data and code availability are available at https://doi.org/10.1038/s41587020-0422-6.

Received: 6 December 2018; Accepted: 10 January 2020; Published online: 10 February 2020

\section{References}

1. Bowers, R. M. et al. Minimum information about a single amplified genome (MISAG) and a metagenome-assembled genome (MIMAG) of bacteria and archaea. Nat. Biotechnol. 35, 725-731 (2017).

2. Kang, D. D., Froula, J., Egan, R. \& Wang, Z. MetaBAT, an efficient tool for accurately reconstructing single genomes from complex microbial communities. PeerJ 3, e1165 (2015).

3. Forster, S. C. et al. A human gut bacterial genome and culture collection for improved metagenomic analyses. Nat. Biotechnol. 37, 186-192 (2019).

4. Nayfach, S., Shi, Z. J., Seshadri, R., Pollard, K. S. \& Kyrpides, N. Novel insights from uncultivated genomes of the global human gut microbiome. Nature 568, 505-510 (2019). 
5. Bishara, A. et al. High-quality genome sequences of uncultured microbes by assembly of read clouds. Nat. Biotechnol. 36, 1067-1075 (2018).

6. Pendleton, M. et al. Assembly and diploid architecture of an individual human genome via single-molecule technologies. Nat. Methods 12, 780-786 (2015).

7. Stewart, R. D. et al. Compendium of 4,941 rumen metagenome-assembled genomes for rumen microbiome biology and enzyme discovery. Nat. Biotechnol. 37, 953-961 (2019).

8. Bertrand, D. et al. Hybrid metagenomic assembly enables high-resolution analysis of resistance determinants and mobile elements in human microbiomes. Nat. Biotechnol. 37, 937-944 (2019).

9. Branton, D. \& Deamer, D. Nanopore Seqeuncing (World Scientific, 2019).

10. Bankevich, A. et al. SPAdes: a new genome assembly algorithm and its applications to single-cell sequencing. J. Comput. Biol. 19, 455-477 (2012).

11. Mikheenko, A., Saveliev, V. \& Gurevich, A. MetaQUAST: evaluation of metagenome assemblies. Bioinformatics 32, 1088-1090 (2015). btv697.

12. Albertsen, M. et al. Genome sequences of rare, uncultured bacteria obtained by differential coverage binning of multiple metagenomes. Nat. Biotechnol. 31, 533-538 (2013).

13. Parks, D. H., Imelfort, M., Skennerton, C. T., Hugenholtz, P. \& Tyson, G. W. CheckM: assessing the quality of microbial genomes recovered from isolates, single cells, and metagenomes. Genome Res. 25, 1043-1055 (2015).

14. Chen, L.-X., Anantharaman, K., Shaiber, A., Murat Eren, A. \& Banfield, J. F. Accurate and complete genomes from metagenomes. Preprint at bioRxiv https://doi.org/10.1101/808410 808410 (2019).

15. Gupta, V. K., Chaudhari, N. M., Iskepalli, S. \& Dutta, C. Divergences in gene repertoire among the reference prevotella genomes derived from distinct body sites of human. BMC Genomics 16, 153 (2015).
16. Seemann, T. Prokka: rapid prokaryotic genome annotation. Bioinformatics 30 2068-2069 (2014).

17. Pasolli, E. et al. Extensive unexplored human microbiome diversity revealed by over 150,000 genomes from metagenomes spanning age, geography, and lifestyle. Cell 176, 649-662.e20 (2019).

18. Zeevi, D. et al. Structural variation in the gut microbiome associates with host health. Nature 568, 43-48 (2019).

19. De Filippis, F. et al. Distinct genetic and functional traits of human intestinal Prevotella copri strains are associated with different habitual diets. Cell Host Microbe 25, 444-453.e3 (2019).

20. Beaulaurier, J. et al. Metagenomic binning and association of plasmids with bacterial host genomes using DNA methylation. Nat. Biotechnol. 36, 61-69 (2018).

Publisher's note Springer Nature remains neutral with regard to jurisdictional claims in published maps and institutional affiliations.

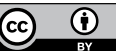

Open Access This article is licensed under a Creative Commons

Attribution 4.0 International License, which permits use, sharing, adaptation, distribution and reproduction in any medium or format, as long as you give appropriate credit to the original author(s) and the source, provide a link to the Creative Commons license, and indicate if changes were made. The images or other third party material in this article are included in the article's Creative Commons license, unless indicated otherwise in a credit line to the material. If material is not included in the article's Creative Commons license and your intended use is not permitted by statutory regulation or exceeds the permitted use, you will need to obtain permission directly from the copyright holder. To view a copy of this license, visit http://creativecommons. org/licenses/by/4.0/.

(C) The Author(s) 2020 


\section{Methods}

Subject recruitment. Two healthy adult volunteers were recruited at Stanford University under an IRB-approved protocol (principal investigator, A. Bhatt). Ten healthy adults were recruited at Stanford University as a part of one of two IRB-approved protocols for tissue biobanking (PIs, A. Bhatt and V. Henderson) Informed consent was obtained for all human subjects. Animal stool samples were collected from a Great Pyrenees housepet dog and an 8-10-week-old female $\mathrm{Balb} / \mathrm{c}(\mathrm{J})$ mouse obtained from the Jackson Laboratory. The discarded mouse stool samples were obtained as a part of an approved laboratory animal use protocol (PI, S. Artandi). We have complied with all relevant ethical regulations.

Sample processing. Stool samples were placed at $4{ }^{\circ} \mathrm{C}$ immediately on collection, and processed for storage at $-80^{\circ} \mathrm{C}$ the same day. Stool samples were aliquoted into $2-\mathrm{ml}$ cryovial tubes with no preservative. Samples were stored at $-80^{\circ} \mathrm{C}$ until extraction.

Stool DNA extraction. Short-read and read-cloud libraries were prepared as previously described ${ }^{5}$. Briefly, DNA was extracted from samples P1 and P2-A with the Qiagen Stool Mini kit using standard bead-beating mechanical lysis. For read-cloud libraries, this was then size selected at $10 \mathrm{kbp}$ with a BluePippin (Sage Science)

For HMW extraction, approximately $0.7 \mathrm{~g}$ of frozen stool was aliquoted into 2-ml Eppendorf tubes (Eppendorf) with a 4-mm biopsy punch (Integra Miltex and suspended in $500 \mu \mathrm{l}$ of PBS (Fisher Scientific) with brief gentle vortexing. Then, $5 \mu \mathrm{l}$ of lytic enzyme solution (Qiagen) and, for the mock mixture and stool samples from the ten subject healthy adult cohort, $10 \mu \mathrm{l}$ of MetaPolyzyme (Sigma Aldrich; reconstituted in $750 \mu \mathrm{l}$ of PBS) was added and the samples were mixed by gentle inversion six times, then incubated for $1 \mathrm{~h}$ at $37^{\circ} \mathrm{C}$. Next, $12 \mu \mathrm{lof} 20 \%(\mathrm{w} / \mathrm{v})$ SDS (Fisher Scientific) was added with approximately $100 \mu \mathrm{l}$ of vacuum grease (DowCorning) functioning as phase lock gel. Then, $500 \mu \mathrm{l}$ of phenol-chloroform isoamyl alcohol at pH 8 (Fisher Scientific) was added, samples were gently vortexed for $5 \mathrm{~s}$ and centrifuged at $10,000 \mathrm{~g}$ for $5 \mathrm{~min}$ with a Legend Micro 21 microcentrifuge (Fisher Scientific). The aqueous phase was then decanted into a new 2-ml tube.

Next, DNA was precipitated with $90 \mu \mathrm{l}$ of $3 \mathrm{M}$ sodium acetate (Fisher Scientific) and $500 \mu \mathrm{l}$ of isopropanol (Fisher Scientific) for $10 \mathrm{~min}$ at room temperature. After inverting three times slowly, samples were incubated at room temperature for $10 \mathrm{~min}$, then centrifuged for $10 \mathrm{~min}$ at $10,000 \mathrm{~g}$. The supernatant was removed and the pellet was washed twice with freshly prepared $80 \%(\mathrm{v} / \mathrm{v})$ ethanol (Fisher Scientific). The pellet was then air dried with heating for $10 \mathrm{~min}$ at $37^{\circ} \mathrm{C}$ or until the pellet was matte in appearance, and then resuspended in $100 \mu \mathrm{l}$ of nuclease-free water (Ambion, Thermo Fisher Scientific). Next, $1 \mathrm{ml}$ of Qiagen buffer G2, $4 \mu \mathrm{l}$ of Qiagen RNase A at $100 \mathrm{mg} \mathrm{ml}^{-1}$ and $25 \mu \mathrm{l}$ of Qiagen Proteinase $\mathrm{K}$ were added, the samples were then gently inverted three times, and then were incubated for $90 \mathrm{~min}$ at $56^{\circ} \mathrm{C}$. After the first $30 \mathrm{~min}$, pellets were dislodged by a single gentle inversion.

One Qiagen Genomic-tip 20/G column per sample was equilibrated with $1 \mathrm{ml}$ of Qiagen buffer QBT and allowed to empty by gravity flow. Samples were gently inverted twice, applied to columns and allowed to flow through. Three stool extractions were combined per column. Columns were then washed with $3 \mathrm{ml}$ of Qiagen buffer QC, then DNA was eluted with $1 \mathrm{ml}$ of Qiagen buffer QF prewarmed to $56^{\circ} \mathrm{C}$. Eluted DNA was then precipitated by addition of $700 \mu \mathrm{l}$ of isopropanol followed by inversion and centrifugation for $15 \mathrm{~min}$ at $10,000 \mathrm{~g}$. The supernatant was carefully removed by pipette and pellets were washed with $1 \mathrm{ml}$ of $80 \%$ (v/v) ethanol. Residual ethanol was removed by air drying $10 \mathrm{~min}$ at $37^{\circ} \mathrm{C}$. This was followed by resuspension of the pellet in $100 \mu \mathrm{l}$ of water overnight at $4{ }^{\circ} \mathrm{C}$ without agitation of any kind

DNA was then size selected with a modified SPRI bead protocol as described in ref. ${ }^{21}$, with minor modifications: beads were added at $0.9 \times$, and eluted DNA was resuspended in $50 \mu \mathrm{l}$ of water. The concentration, purity and fragment size distribution of extracted DNA was then quantified with the Qubit fluorometer (Thermo Fisher Scientific), Nanodrop (Thermo Fisher Scientific) and TapeStation 2200 or 4200 (Agilent Technologies), respectively (Supplementary Table 1). All steps were carried out at room temperature unless otherwise stated.

Defined mixture DNA extraction. For the defined bacterial mixture, two aliquots of lyophilized cells were obtained (item MSA-2006, ATCC) and resuspended in $500 \mu \mathrm{l}$ of PBS. With $2.4 \times 10^{8}$ cells provided, this gives a total theoretical mass of available DNA of approximately $2.2 \mu \mathrm{g}$ before size selection, with $1 \mu \mathrm{g}$ of sizeselected HMW DNA required for long-read library preparation. Due to the extremely limited DNA available, as well as the lower purification requirements of this sample compared to stool, the HMW DNA extraction protocol was carried out as described above omitting the digestion and Genomic-tip purification steps. Samples were then quantified as above.

Sequencing. Extracted DNA samples were prepared for long-read sequencing with the Oxford Nanopore Technologies (ONT) Ligation library preparation kit according to the manufacturer's standard protocol with the addition of continuous gentle mixing during the ligation incubation step. Stool libraries were sequenced with the ONT MinION sequencer using rev C R9.4 flow cells, allocating one flowcell per sample. The defined bacterial mixture and each of the ten healthy adult samples was allocated one revolution of a D R9.4 flowcell. The sequencer was controlled with the MinKNOW v.2.2.12 software running on a MacBook Pro (model A1502, Apple), with data stored to a Vectotech 2Tb solid-state hard drive. Sequencing runs were scheduled for $48-60 \mathrm{~h}$, and allowed to run until fewer than ten pores remained functional. After sequencing, data were uploaded to the Stanford Center for Genomics computational cluster for analysis. Stool sample short-read libraries were prepared and sequenced as described previously ${ }^{5}$. The 250-bp paired-end ATCC mixture short-read data were supplied by OneCodex. The PacBio long-read library was prepared and sequenced with one SMRT cell on a Sequel sequencing instrument (Pacific Biosciences) according to the manufacturer's standard protocol by the University of California Davis Genome DNA Technologies Core.

Sequence assembly. Lathe generates raw basecalled data using Guppy v.2.3.5 and produces two subassemblies in two separate runs with either Flye v.2.4.2 with the -meta parameter, or Canu v.1.8 using the -nanopore preset parameter ${ }^{22}$. In either case, the two runs differ by the estimated genomeSize parameter, provided as 50 and $100 \mathrm{~m}$ for Canu, or 100 and $250 \mathrm{~m}$ for Flye. The two separate assemblies are then merged with quickmerge v.0.40 (ref. ${ }^{23}$ ) with parameters $-1 \mathrm{~m} 40000-\mathrm{c}$ 5 -hco 10, polished with either Racon v.1.3.2 (ref. ${ }^{24}$ ) and Medaka v.0.6.1 (ref. ${ }^{25}$ ) or a parallelized version of Pilon v.1.22 (ref. ${ }^{26}$ ) for long- or short-read consensus refinement, respectively, then circularized. To parallelize Pilon, necessary for application to metagenomic assemblies, reference sequences are divided into 100$\mathrm{kb}$ segments, short reads aligned to each segment downsampled to $50 \times$ coverage depth and Pilon is then used to detect errors within the reference and read subset. These errors are then aggregated across all subset runs and used to generate a refined consensus with bcftools v.1.9-107 (ref. ${ }^{27}$ ). Errors found in homopolymers were identified with an in-house script. Sequences were then binned and annotated as previously described ${ }^{5}$.

Lathe applies two methods to evaluate circularity and precisely locate the genome wrap-around point in single-contig genomes, which we term circularization. The first method detects over-circularized contigs, which are those genome contigs assembled beyond the wrap-around point of the circular chromosome resulting in redundant sequences at the contig termini. This is done by self-alignment with nucmer v.3.1 (ref. ${ }^{28}$ ) followed by analysis by a custom script. The second attempts to assemble a contig spanning across the two ends of a candidate genome. This is done by collecting reads aligning to the termini of the candidate genome, assembling with Canu, then aligning the resultant spanning contig to the candidate genome and testing for alignment consistent with a closed circular genome. The last method is conceptually similar to an existing approach ${ }^{29}$, but differs primarily in its parallelized implementation of spanning contig assembly and detection, which achieves a large reduction in runtime.

To detect misassemblies, Lathe searches for locations in the assembly spanned across by one or zero long reads, indicating either a total lack of support for true contiguity or support from only a single possibly chimeric read. It does this by breaking the genome into windows smaller than the average read length, then measuring coverage within each window from reads spanning the entire window. With no misassembly, an assembly produced from a given readset will have all windows spanned by the assembled reads. A misassembly within a given window will cause read alignments to be soft-clipped at the misassembly breakpoint, preventing read alignments from spanning across the breakpoint and therefore the window. Contigs are then broken at identified misassembled sites before final output generation.

Lathe was compared to the long-read assemblers miniasm v.0.2 (ref. ${ }^{30}$ ) Ra v.0.2.1 (ref. ${ }^{31}$ ), wtdbg2 v.2.2 (ref. ${ }^{32}$ ) and Flye v.2.4.2 (ref. ${ }^{33}$ ), as well as the hybrid assemblers OPERA-MS ${ }^{8}$ and hybridSPAdes v.3.13.0 (ref. ${ }^{34}$ ), for the two healthy human stool (samples P1 and P2-A) (Supplementary Table 4). For hybrid approaches, we supplemented the long-read datasets with the short-read datasets previously generated for these samples ${ }^{5}$. Default parameters were used for all assembly approaches. Assembly N50, total size and longest contig were calculated with Quast v.5.0.0 (ref. ${ }^{11}$ ). Lathe can be found at https://github.com/bhattlab/lathe/.

Genome analysis. Binning was performed and evaluated as previously described ${ }^{5}$. Genomes were compared to reference sequences by alignment with Mummer ${ }^{28}$. Long and short reads were taxonomically classified with $\mathrm{Kraken}^{35}$, and Shannon diversity was calculated with vegan ${ }^{36}$. We note that classifiers developed for short reads of uniform length do not correct for the variable read length of long reads, counting relative read counts and not relative number of bases sequenced, which may slightly bias relative abundance results. rRNA presence was determined with Barrnap v.0.9 $\left(\right.$ ref. ${ }^{37}$ ). Gene count and insertion sequence transposase count was determined with Prokka v.1.13.3 (ref. ${ }^{16}$ ). For P. copri, additional insertion sequence locations were determined by mapping previously identified $P$. copri insertion sequences ${ }^{5}$ to the circular genome. Putative phage regions were identified with PHASTER ${ }^{38}$. Figures were generated with ggplot2 v.3.2.1 (ref. ${ }^{39}$ ). Downstream analysis workflows can be found at https://github.com/bhattlab/metagenomics_workflows/.

Novel species identification. Classifications for the unknown genome assembled in sample P1 and shown in Fig. 2 and Table 1 as F. prausnitzii were attempted with BLAST v.2.9.0 (ref. ${ }^{40}$ ) against the NCBI Genbank database ${ }^{41}$, 16S identification 
with Barrnap v.0.9 (ref. ${ }^{37}$ ) and BLAST against the Ribosomal Database Project database ${ }^{42}$ and NCBI 16 S Archaeal and Bacterial database, and Kraken2 classification $^{35}$. Genome sequences were compared to the assembled genome draft by alignment and post-processing with mummer ${ }^{28}$.

Reporting Summary. Further information on research design is available in the Nature Research Reporting Summary linked to this article.

\section{Data availability}

All sequence data, whole metagenome assemblies and individual completed genomes can be found at the NCBI BioProject under accession code PRJNA508395.

\section{Code availability}

Lathe is available at https://github.com/bhattlab/lathe. Version 1.0 was used for analyses in this paper. Binning, annotation and post-processing workflows can be found at https://github.com/bhattlab/metagenomics_workflows.

\section{References}

21. Nagar, R. \& Schwessinger, B. DNA size selection $(>3-4 \mathrm{~kb})$ and purification of DNA using an improved homemade SPRI beads solution. v.1. Protocols.io https://doi.org/10.17504/protocols.io.n7hdhj6 (2018).

22. Koren, S. et al. Canu: scalable and accurate long-read assembly via adaptive k-mer weighting and repeat separation. Genome Res. 27, 722-736 (2017).

23. Chakraborty, M., Baldwin-Brown, J. G., Long, A. D. \& Emerson, J. J. Contiguous and accurate de novo assembly of metazoan genomes with modest long read coverage. Nucleic Acids Res. 44, e147 (2016).

24. Vaser, R., Sović, I., Nagarajan, N. \& Šikić, M. Fast and accurate de novo genome assembly from long uncorrected reads. Genome Res. 27, 737-746 (2017)

25. Medaka 0.3.0 Documentation (Oxford Nanopore Technologies, 2018); https://nanoporetech.github.io/medaka/index.html

26. Walker, B. J. et al. Pilon: an integrated tool for comprehensive microbial variant detection and genome assembly improvement. PLOS ONE 9, e112963 (2014).

27. Danecek, P. Others. bcftools-utilities for variant calling and manipulating vcfs and bcfs (GitHub, 2015).

28. Delcher, A. L., Salzberg, S. L. \& Phillippy, A. M. Using MUMmer to identify similar regions in large sequence sets. Curr. Protoc. Bioinformatics 00 , 10.3.1-10.3.18 (2003).

29. Hunt, M. et al. Circlator: automated circularization of genome assemblies using long sequencing reads. Genome Biol. 16, 294 (2015).

30. Li, H. Minimap and miniasm: fast mapping and de novo assembly for noisy long sequences. Bioinformatics 32, 2103-2110 (2016).

31. Vaser, R. ra v0.2.1 (Github).

32. Ruan, J. \& Li, H. Fast and accurate long-read assembly with wtdbg2. Nat. Methods 37, 937-944 (2019).

33. Kolmogorov, M. \& Yuan, J. \& Lin, Y. \& Pevzner, P. A. Assembly of long, error-prone reads using repeat graphs. Nat. Biotechnol. 37, 540-546 (2019).
34. Antipov, D., Korobeynikov, A., McLean, J. S. \& Pevzner, P. A. hybridSPAdes: an algorithm for hybrid assembly of short and long reads. Bioinformatics 32, 1009-1015 (2016).

35. Wood, D. \& Salzberg, S. Kraken: ultrafast metagenomic sequence classification using exact alignments. Genome Biol. 15, R46 (2014).

36. Dixon, P. VEGAN, a package of R functions for community ecology. J. Veg. Sci. 14, 927-930 (2003).

37. Seemann, T. barrnap v2.2 (Github).

38. Arndt, D. et al. PHASTER: a better, faster version of the PHAST phage search tool. Nucleic Acids Res. 44, W16-W21 (2016).

39. Wickham, H. ggplot2: Elegant Graphics for Data Analysis (Springer Science \& Business Media, 2009).

40. Altschul, S. F., Gish, W., Miller, W., Myers, E. W. \& Lipman, D. J. Basic local alignment search tool. J. Mol. Biol. 215, 403-410 (1990).

41. Benson, D. A. et al. GenBank. Nucleic Acids Res. 41, D36-D42 (2013).

42. Maidak, B. L. et al. The RDP (Ribosomal Database Project). Nucleic Acids Res. 25, 109-111 (1997).

\section{Acknowledgements}

We acknowledge G. Sherlock and the members of the Bhatt laboratory for experimental advice and assistance, $H$. Tang for statistical advice, C. Severyn for helpful review of the manuscript, P. Neuhoefer, J. Ribado and R. Patel for providing mouse stool and performing extraction and quantification, OneCodex for providing short-read sequence data for the bacterial mixture and J. Hanks (Griznog) for computational assistance. E.L.M. was supported by the National Science Foundation Graduate Research Fellowship no. DGE-114747. D.G.M. was supported by the Stanford Graduate Fellowships in Science and Engineering program. This work was supported by the Damon Runyon Clinical investigator award and grant no. NIH R01AI148623 to A.S.B. and grant no. NIH P30 AG047366, which supports the Stanford ADRC. Computational work was supported by NIH S10 Shared Instrumentation grant no. 1S10OD02014101 and by NIH grant no. P30 CA124435, which supports the Genetics Bioinformatics Service Center, a Stanford Cancer Institute Shared Resource.

\section{Author contributions}

E.L.M and A.S.B. conceived the study. E.L.M developed the extraction method and Lathe workflow and performed extraction, sequencing and assembly on all samples. A.S.B., E.L.M. and D.G.M. carried out all analyses, wrote the manuscript and generated figures. All authors read and approved the final manuscript.

\section{Competing interests}

The authors declare no competing interests.

\section{Additional information}

Supplementary information is available for this paper at https://doi.org/10.1038/ s41587-020-0422-6.

Correspondence and requests for materials should be addressed to A.S.B.

Reprints and permissions information is available at www.nature.com/reprints. 


\section{Reporting Summary}

Nature Research wishes to improve the reproducibility of the work that we publish. This form provides structure for consistency and transparency in reporting. For further information on Nature Research policies, see Authors \& Referees and the Editorial Policy Checklist.

\section{Statistics}

For all statistical analyses, confirm that the following items are present in the figure legend, table legend, main text, or Methods section.

$\mathrm{n} / \mathrm{a}$ Confirmed

$\bigotimes$ The exact sample size $(n)$ for each experimental group/condition, given as a discrete number and unit of measurement

\A statement on whether measurements were taken from distinct samples or whether the same sample was measured repeatedly

The statistical test(s) used AND whether they are one- or two-sided

Only common tests should be described solely by name; describe more complex techniques in the Methods section.

Х $\square$ A description of all covariates tested

Х $\square$ A description of any assumptions or corrections, such as tests of normality and adjustment for multiple comparisons

Х A full description of the statistical parameters including central tendency (e.g. means) or other basic estimates (e.g. regression coefficient)

$\triangle$ AND variation (e.g. standard deviation) or associated estimates of uncertainty (e.g. confidence intervals)

$\bigvee$ For null hypothesis testing, the test statistic (e.g. $F, t, r$ ) with confidence intervals, effect sizes, degrees of freedom and $P$ value noted Give $P$ values as exact values whenever suitable.

Х $\square$ For Bayesian analysis, information on the choice of priors and Markov chain Monte Carlo settings

Х $\square$ For hierarchical and complex designs, identification of the appropriate level for tests and full reporting of outcomes

$\square$ Estimates of effect sizes (e.g. Cohen's d, Pearson's $r$ ), indicating how they were calculated

Our web collection on statistics for biologists contains articles on many of the points above.

\section{Software and code}

Policy information about availability of computer code

Data collection

MinKNOW v2.2.12, Guppy v2.3.5 were used to collect and base-call collected DNA sequencing data from the ONT MinION platform.

Data analysis

BLAST v2.9.0, Canu v1.8, Pilon v1.22, CheckM v1.0.12, Racon v1.3.2, Medaka v0.6.1, mummer v3.23, miniasm v0.2, SPAdes v3.13.0, hybridSPAdes v3.13.0, wtdbg2 v2.2, Ra v0.2.1, Flye v2.4.2, quickmerge v0.40, OPERA-MS, Quast v5.0.0, Prokka v1.13.3, Barrnap v0.9, nucmer v3.1, bcftools v1.9-107, ggplot2 v3.2.1, PHASTER, 2.0.8, and Snakemake v5.4.0 were used to analyze the data used in this study. In addition, custom workflows and scripts used in this study can be found at https://github.com/elimoss/metagenomics_workflows.

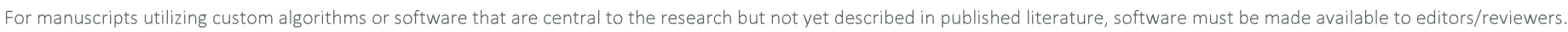
We strongly encourage code deposition in a community repository (e.g. GitHub). See the Nature Research guidelines for submitting code \& software for further information.

\section{Data}

Policy information about availability of data

All manuscripts must include a data availability statement. This statement should provide the following information, where applicable:

- Accession codes, unique identifiers, or web links for publicly available datasets

- A list of figures that have associated raw data

- A description of any restrictions on data availability

All sequence data, whole metagenome assemblies and individual completed genomes can be found at NCBI BioProject under accession PRJNA508395. 
Please select the one below that is the best fit for your research. If you are not sure, read the appropriate sections before making your selection.

$\bigotimes$ Life sciences $\quad \square$ Behavioural \& social sciences $\square$ Ecological, evolutionary \& environmental sciences

For a reference copy of the document with all sections, see nature.com/documents/nr-reporting-summary-flat.pdf

\section{Life sciences study design}

All studies must disclose on these points even when the disclosure is negative.

Sample size No sample size calculation was performed, as this study examines DNA sequencing and assembly methods on previously published comparison materials.

Data exclusions No data were excluded from analysis

Replication The analytical approaches used in this paper are entirely deterministic, and the same assembly result can be obtained from the input data by re-running the published workflow.

Randomization There are no experimental groups in this study, so no randomization was needed.

Blinding There are no experimental groups in this study, so no blinding was needed.

\section{Reporting for specific materials, systems and methods}

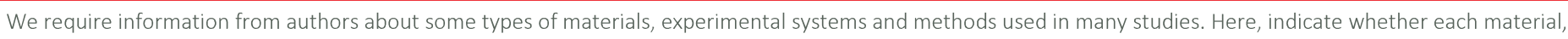

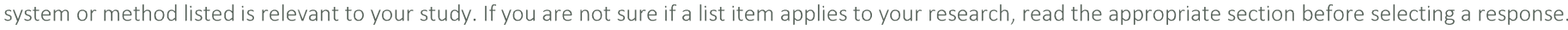

\begin{tabular}{|c|c|}
\hline$n / a$ & Involved in the study \\
\hline Х & Antibodies \\
\hline Х & Eukaryotic cell lines \\
\hline$\bigotimes$ & $\square$ Palaeontology \\
\hline$\square$ & $\bigotimes$ Animals and other organisms \\
\hline \begin{tabular}{|c} 
\\
\end{tabular} & $\bigotimes$ Human research participants \\
\hline Х & $\square$ Clinical data \\
\hline
\end{tabular}

\begin{tabular}{|c|c|}
\hline$n / a$ & Involved in the study \\
\hline Х & ChIP-seq \\
\hline Х & Flow cytometry \\
\hline Х & $\square$ MRI-based neuroimaging \\
\hline
\end{tabular}

\section{Animals and other organisms}

Policy information about studies involving animals; ARRIVE guidelines recommended for reporting animal research

Laboratory animals

Wild animals

Field-collected samples

Ethics oversight
Stool samples from a mouse were used in this study. The mouse samples were provided by Patrick Neuhoefer from the laboratory of Steven Artandi.

None

Discarded stool samples were collected from a domestic house-pet dog for this study.

All mouse samples were collected in accordance with an approved animal use protocol (Stanford University) overseen by the Stanford APLAC (Laboratory Animal Care) committee. The house-pet dog stool samples were discarded samples, the animal was put at minimal risk by this sample collection and thus did not require ethics approval for use.

Note that full information on the approval of the study protocol must also be provided in the manuscript.

\section{Human research participants}

Policy information about studies involving human research participants

Population characteristics

Recruitment
Adult participants were recruited from at Stanford University under one of two approved IRB protocols. Because this is a sequencing method development study, no covariates were used for selection.

Human subjects were invited to participate in one of two observational biobanking studies through informational flyers that were posted in various public places. Interested volunteers were then consented by the PI or study staff of one of the two 
studies for participation in this study. The advertising flyers, protocol, and consent documents were all approved by the Stanford IRB (Study \#42043; PI: Bhatt; Study \#33727, PI: Henderson)

\section{Ethics oversight}

Stanford Institutional Review Board

Note that full information on the approval of the study protocol must also be provided in the manuscript. 